Service social

\title{
Effets psychosociaux des activités visant à soutenir les jeunes adultes en situation de handicap : une recension des écrits
}

\section{Sabrina Faleschini et Normand Boucher}

Volume 62, numéro 2, 2016

URI : https://id.erudit.org/iderudit/1038575ar

DOI : https://doi.org/10.7202/1038575ar

Aller au sommaire du numéro

\section{Éditeur(s)}

École de travail social et de criminologie de l’Université Laval

ISSN

1708-1734 (numérique)

Découvrir la revue

Citer cet article

Faleschini, S. \& Boucher, N. (2016). Effets psychosociaux des activités visant à soutenir les jeunes adultes en situation de handicap : une recension des écrits. Service social, 62(2), 33-48. https://doi.org/10.7202/1038575ar
Résumé de l'article

Contexte : À la fin de l'adolescence, les jeunes en situation de handicap vivent une transition entre la sortie du système scolaire et l'adaptation à la vie adulte. Certaines activités semblent promouvoir le développement d'habiletés personnelles pendant cette période de développement. Objectif : Documenter les effets psychosociaux des activités visant à soutenir les jeunes adultes en situation de handicap. Méthode : Une revue de littérature a été réalisée dans les bases de données PubMed, ERIC, CINAHL et Social Work. Résultats : Un total de 35 articles a été conservé. Cinq types d'activités ont été abordés : l'activité physique, le travail, les loisirs, l'école et la thérapie par la musique. Les activités ont des conséquences sur la qualité de vie générale, et plus particulièrement sur la perception de soi et les relations interpersonnelles. Conclusion : Les activités ont généralement des effets psychosociaux positifs. Toutefois il importe de prendre en compte le contexte de leur réalisation, par exemple offrir un contexte d'activités compétitives plutôt que récréatives, afin de mettre en place des conditions optimales au développement d’habiletés. 


\title{
Effets psychosociaux des activités visant à soutenir les jeunes adultes en situation de handicap : une recension des écrits
}

\author{
FALESCHINI, Sabrina \\ Candidate au doctorat en psychologie \\ École de psychologie \\ Université Laval \\ BOUCHER, Normand \\ Chercheur \\ CIRRIS
}

\section{RÉSUMÉ}

Contexte : À la fin de l'adolescence, les jeunes en situation de handicap vivent une transition entre la sortie du système scolaire et l'adaptation à la vie adulte. Certaines activités semblent promouvoir le développement d'habiletés personnelles pendant cette période de développement. Objectif : Documenter les effets psychosociaux des activités visant à soutenir les jeunes adultes en situation de handicap. Méthode : Une revue de littérature a été réalisée dans les bases de données PubMed, ERIC, CINAHL et Social Work. Résultats : Un total de 35 articles a été conservé. Cinq types d'activités ont été abordés : l'activité physique, le travail, les loisirs, l'école et la thérapie par la musique. Les activités ont des conséquences sur la qualité de vie générale, et plus particulièrement sur la perception de soi et les relations interpersonnelles. Conclusion : Les activités ont généralement des effets psychosociaux positifs. Toutefois il importe de prendre en compte le contexte de leur réalisation, par exemple offrir un contexte d'activités compétitives plutôt que récréatives, afin de mettre en place des conditions optimales au développement d'habiletés.

Mots-clés : activités, handicap, participation

\begin{abstract}
Introduction: In late adolescence, young people with disabilities live a transition between end of school and adaptation to adulthood. Some activities appear to promote the development of skills that foster the transition to adulthood. Objective: To document the psychosocial effects and the context of activities aiming to support young adults with disabilities. Methods: A literature review was conducted in four databases: PubMed, ERIC, CINAHL and Social Work. Results: A total of 35 articles were selected. Five types of activities were discussed: physical activity, work, recreation, school and music therapy. The activities have an impact on three indicators of general quality of life, self-perception and interpersonal relationships. Conclusion: The activities generally have positive psychosocial effects; however it is important to consider the context of their implementation in order to establish optimal conditions for critical skills development to prepare young people in transition to adult life.
\end{abstract}

Keywords : Activities, disabilities, participation 


\section{INTRODUCTION}

La fin de la période de l'adolescence représente un jalon important du développement de l'identité chez les jeunes en voie de devenir adultes. À ce moment, les perceptions qu'ils possèdent concernant leur propre personne deviennent plus unifiées leur permettant une plus grande autonomie (Smetana, 2011). Ce phénomène d'autonomisation de la pensée va de pair avec le déploiement d'une autonomie plus générale dans la vie du jeune, visant la prise de contrôle et la responsabilité de sa propre vie. Collins et O'Mahony (2015) rapportent que l'autonomie est valorisée chez les personnes ayant une déficience physique et va influencer leur choix d'activités occupationnelles.

Le Processus de production du handicap (PPH) est une approche proposant un modèle conceptuel visant à documenter les causes et conséquences des atteintes à l'intégrité d'une personne (Fougeyrollas, 2010, p. 152). Selon cette approche, la présence ou l'absence d'une incapacité est fonction de l'environnement, composé d'obstacles et de facilitateurs, et non la responsabilité d'un individu. Le développement d'une autonomie décisionnelle pour le jeune en voie de devenir adulte pourrait agir en lui permettant de vivre de nouvelles expériences et surtout le mener à faire des choix et à prendre des décisions sur une base davantage volontaire, en fonction de ses goûts et de ses valeurs. II pourrait dépasser les restrictions que la société lui impose du fait de son handicap, telles qu'une limitation à la participation sociale, à son épanouissement personnel et à ses droits comme personne (Fougeyrollas, 2010, p. 22). En effet, comme le mentionne Fougeyrollas (2010, p. 22) : « ce sont les structures sociales et économiques de sociétés particulières qui, par des processus institutionnalisés d'oppression, d'exclusion, de dévalorisation, d'invalidation, créent le handicap. »

Au Québec, 9,5\% des personnes âgées de 15 ans et plus vivraient avec une incapacité (Statistique Canada, 2013). Ces personnes vivent généralement un processus de transition important les menant de la sortie du système scolaire à une vie d'adulte accomplie. Cette période demande à la personne une adaptation particulière étant donné les difficultés singulières auxquelles elle fait face et elle est marquée par une interruption des services qui étaient jusqu'alors offerts aux adolescents. Dans cette période délicate du développement, ces conséquences peuvent avoir des répercussions à long terme qui seraient dommageables pour ces adultes en devenir.

Les personnes vivant avec une incapacité seraient en moins grande proportion actives sur le marché du travail, vivraient moins d'occasions de socialisation et auraient une moins bonne qualité de vie que les personnes sans incapacité (National Organization on Disability, 2010). II est intéressant de noter que certaines stratégies peuvent être mises en place afin de favoriser un déroulement optimal de la période transition, permettant ainsi aux personnes de vivre une adaptation réussie à la vie adulte ${ }^{1}$. L'Office des personnes handicapées du Québec (OPHQ, 2003) met de l'avant trois orientations possibles que peut prendre la période de transition entre la sortie du système scolaire et la vie adulte. Premièrement, cette période peut être orientée vers un parcours socioprofessionnel, vécue en terme d'aspects particuliers à travailler pour le jeune, comme la préparation à la vie en appartement. Elle peut aussi être dirigée vers la participation à de la formation ou, finalement, prendre la forme d'un travail sur les habiletés personnelles spécifiques qui permettront de préparer adéquatement l'adolescent à sa vie

\footnotetext{
${ }^{1}$ Au Québec, il existe une approche nommée La transition de l'école vers la vie active (TEVA) qui vise à planifier adéquatement la transition vers la vie adulte à l'aide d'un ensemble de services et d'activités permettant d'assurer au jeune un soutien adéquat en fonction de ses besoins et de son projet de vie (Landry et Boucher, 2011).
} 
d'adulte. Ces trois avenues demandent certainement le développement d'habiletés personnelles précises afin d'être réalisées avec succès.

L'OPHQ (2013) note que les activités physiques, sportives ou récréatives ont des retombées positives sur la santé des personnes ayant des incapacités tout en constituant une occasion de participation sociale. En plus des effets positifs sur la santé des personnes, ces activités pourraient également amener des effets psychosociaux bénéfiques notamment en améliorant la qualité de vie des personnes. L'objectif de cet article vise donc à documenter les effets psychosociaux et le contexte de réalisation des activités soutenant les jeunes adultes en situation de handicap.

\section{Méthode}

Une recension des écrits a été effectuée afin de mieux comprendre les effets psychosociaux des activités permettant de soutenir les jeunes adultes en situation de handicap ainsi que leur contexte de réalisation. Dans le cadre de cette recension, une situation de handicap est définie par « une réalisation réduite ou perturbée des habitudes de vie » (Fougeyrollas, 2010, p. 153). Selon Fougeyrollas (2010, p. 159), une habitude de vie correspond à « une activité courante ou un rôle social valorisé par la personne ou son contexte socioculturel selon ses caractéristiques (âge, sexe, identité socioculturelle, etc.). [...] La qualité de réalisation d'une habitude de vie se mesure, à son tour, sur une échelle allant de la situation de pleine participation sociale à la situation de handicap totale ». Les gens désignés par « jeunes adultes » constituent un groupe de personnes âgées entre 16 et 30 ans. II est donc possible que certaines études portent uniquement sur la période de l'adolescence de même que sur l'âge adulte. Pour effectuer cette recension, quatre bases de données ont été consultées en mai et juin 2015 : (1) PubMed, (2) ERIC, (3) CINAHL et (4) Social Work.

Les termes utilisés pour la recherche ont été regroupés en trois thèmes. Ils ont été déterminés avec l'aide de spécialistes en documentation en fonction de l'indexation particulière des bases de données. Ces thèmes vont comme suit : (1) La population à l'étude, désignée par jeunes adultes en situation de handicap, est représentée par des termes reliés à l'incapacité par exemple disabled, intellectual disability ou paraplegia. (2) Les activités sont désignées par des termes comme sports, leisure activities ou work. (3) Les impacts psychosociaux sont désignés par des termes tels que quality of life, self concept ou self-esteem. Les articles doivent avoir été indexés par au moins un mot de chacune des catégories. Des articles pertinents, identifiés à partir de la liste de références des articles consultés ont également été inclus. Les critères d'inclusion sont : (1) L'article est disponible en langue anglaise ou française; (2) Les participants sont en situation de handicap; (3) Le groupe d'âge à l'étude comporte des participants ayant entre 16 et 30 ans; (4) Les études ont été publiées sur une période de 10 ans allant de 2005 à 2015.

\section{Résultats}

Un total de 35 articles a été conservé (voir Figure 1). Cinq types d'activités ont été couverts par les articles: l'activité physique, le travail, les loisirs, l'école et la thérapie par la musique. Les activités semblent avoir des conséquences sur la qualité de vie générale et plus particulièrement sur la perception de soi et les relations interpersonnelles (voir Figure 2). La qualité de vie peut être définie en termes de huit différents domaines pouvant faire l'objet d'une évaluation : le développement personnel, 
le sentiment d'auto-détermination, les relations interpersonnelles, l'inclusion sociale, les droits de la personne, le bien-être émotionnel, physique et matériel (Schalock, Bonham et Verdugo, 2008).

\section{Types d'activités}

\section{Les activités physiques}

La participation à des activités physiques, tant de manière individuelle qu'en groupe, est associée à des impacts psychosociaux positifs sur la qualité de vie des personnes ayant une incapacité physique. La réalisation de ces activités agirait en améliorant la santé mentale, le bien-être et les relations sociales (Nasuti et Temple, 2010). Ces résultats sont observables notamment chez les personnes ayant une amputation alors qu'une revue systématique (Bragaru, Dekker, Geertzen et Dijkstra, 2011) met de l'avant l'augmentation du sentiment de contrôle perçu de même qu'une plus grande acceptation de la déficience physique auprès des personnes. Un programme individualisé d'exercices adaptés, réalisé deux fois par semaine pendant 10 semaines a permis d'améliorer la qualité de vie de personnes ayant une lésion de la moelle épinière (Semerjian et al., 2005). La participation à des activités physiques pendant ses temps libres est associée à une plus grande qualité de vie chez cette population (Sweet, Martin Ginis et Tomasone, 2013).

Il est intéressant de noter que le sport de compétition a également des retombées importantes auprès des personnes ayant une déficience physique. Chez des athlètes ayant une paraplégie, ce contexte a été associé à une amélioration de la qualité de vie générale, de la santé mentale, du sommeil de même qu'à un plus grand sentiment d'efficacité personnelle (Fiorilli et al., 2013; Giacobbi Jr et al. 2008; Martin, 2008; Yazicioglu et al., 2012). De plus, selon Sweet et collaborateurs (2013), deux facteurs pourraient être mis de l'avant pour expliquer la relation entre la participation à des loisirs sportifs et la qualité de vie des personnes ayant une lésion à la moelle épinière : la présence de symptômes dépressifs de même que l'indépendance fonctionnelle des personnes auraient une influence sur cette relation. II est connu que les activités physiques ont un rôle à jouer dans la santé mentale et permettraient d'augmenter l'indépendance fonctionnelle des personnes qui les pratiquent. De fait, selon Dijkers (2005), la participation à des activités physiques représenterait une réussite personnelle en favorisant le développement de l'indépendance fonctionnelle des personnes. Par contre, chez celles participant à des activités physiques préalablement à la conduite de l'étude, il est possible de se demander si ce sont les activités qui améliorent la qualité de vie ou si ce ne serait pas les personnes ayant déjà une qualité de vie plus élevée qui auraient tendance à réaliser des activités physiques (Yazicioglu et al., 2012).

Les activités physiques dans un contexte compétitif ont également des impacts chez les personnes ayant une paralysie cérébrale (Groff, Lundberg et Zabriskie, 2009). Dans cette étude, les participants étaient des athlètes d'élite participant au World Cerebral Palsy Championship aux États-Unis. Les participants rapportent l'expression d'une identité d'athlète ainsi qu'une meilleure perception de leurs compétences sociales suite à leur participation. De plus, selon Groff, Lundberg et Zabriskie (2009), l'adhésion à une identité d'athlète de même que la sévérité de l'incapacité prédiraient l'influence des activités physiques sur la qualité de vie. Une seconde étude (Hutzler, Chacham-Guber et Reiter, 2013) a trouvé un effet de l'activité physique uniquement si celle-ci est réalisée à la manière d'une « intégration inversée ", c'est-à-dire comme une activité réalisée en groupe où la majorité des participants sont des personnes ayant une incapacité et où une minorité est donc sans incapacité. Ce type d'environnement favoriserait tant la coopération que la compétition entre les membres (Brasile, 1992). Selon Brasile, une 
approche intégrée permet à la personne de sortir du cadre de la réadaptation traditionnelle dans lequel elle ne pourrait pas être en compétition sportive avec des personnes n'ayant pas une déficience similaire. De plus, Hutzler, Chacham-Guber et Reiter (2013) mettent en rapport l'importance de la sévérité de l'incapacité avec la participation à des activités chez cette population. Étant donné que la participation à des activités semble varier en fonction du niveau d'habileté fonctionnelle, les personnes vivant des restrictions fonctionnelles sévères semblent moins retirer de bénéfices de leur participation à des sports.

Chez les personnes ayant une déficience intellectuelle, la présence de compétition dans les activités sportives semble avoir des effets qui sont parfois différents. Premièrement, la formation d'une identité d'athlète semble avoir été favorisée auprès des participants des Jeux Olympiques Spéciaux Mondiaux (Werner, 2015). Toutefois, une seconde étude mentionne que la participation régulière à un programme de compétition athlétique réalisée de manière intégrée est liée à une diminution de l'estime de soi s'expliquant par une vision plus réaliste qu'ont les personnes de leur capacité lorsqu'elles sont dans un nouveau système de comparaison sociale (Ninot, Bilard et Delignieres, 2005). II convient donc de prendre en compte le groupe de comparaison auquel les personnes seront confrontées lors de la réalisation des activités afin de pouvoir établir un climat de coopération favorisant les apprentissages et le développement de relations sociales.

Il est possible de constater des résultats équivalents chez des personnes ayant le spina-bifida (Buffart et al., 2009). Dans le cadre de cette étude, les personnes réalisant un niveau élevé d'activités physiques présentent une qualité de vie plus élevée que celles participant à un niveau plus faible d'activités. À l'instar de Hutzler, Chacham-Guber et Reiter (2013), les auteurs ont constaté que les personnes ayant une moins grande mobilité percevraient une qualité de vie plus basse que celles ayant une plus grande indépendance fonctionnelle. Ceci est également observé chez les personnes ayant la sclérose en plaques où Sung et al. (2012) ont démontré que l'exercice physique serait un des médiateurs partiels de la relation entre l'incapacité et la qualité de vie. Toutefois, si l'exercice physique peut expliquer en partie la relation entre l'incapacité et la qualité de vie, le niveau de sévérité de l'incapacité reste un déterminant majeur du niveau de qualité de vie perçu par les participants.

Des bénéfices des activités physiques sont constatés également chez les personnes ayant une déficience intellectuelle. Une revue systématique (Bartlo et Klein, 2011) mentionne que ces personnes expriment une meilleure qualité de vie suite à la participation à une intervention visant à faire vivre des activités physiques. L'augmentation du niveau de qualité de vie pourrait s'expliquer par le fait que les activités physiques favoriseraient une meilleure perception de leur bien-être de même qu'une plus grande performance fonctionnelle (Tsimaras et Fotiadou, 2004). Il est également possible de constater une réduction significative des symptômes dépressifs lors d'une intervention de 12 semaines visant à faire vivre aux personnes des séances d'activités physiques variées en groupe (Carraro et Gobbi, 2014). Une revue systématique rapporte une diminution des symptômes agressifs à la suite d'une intervention visant à mettre en place des séances d'exercices, tant en groupe qu'individuelles (Ogg-Groenendaal, Hermans et Claessens, 2014). Les auteurs ont noté que la réduction des symptômes agressifs pourrait être liée au sentiment d'accomplissement ressenti par les personnes.

Peu d'études se sont intéressées aux impacts des activités physiques sur la qualité de vie chez les personnes ayant une incapacité visuelle ou auditive. Une seule étude a été répertoriée (Di Cagno et al., 2013) et mentionne que les personnes ayant une déficience visuelle qui participent à des ligues de torball compétitif au niveau national, rapportent une meilleure santé psychologique, un plus grand bien- 
être et une plus grande participation sociale que celles ne participant pas à l'activité. Les auteurs notent que leur participation à des activités physiques pourrait favoriser leur résilience.

En plus d'une augmentation générale de la qualité de vie des personnes pratiquant des activités physiques, des auteurs notent un effet bénéfique sur la perception de soi des individus. Cette perception de soi positive se manifeste notamment par un plus grand sentiment d'indépendance et une augmentation de la confiance en soi chez les athlètes ayant une déficience physique comparativement aux personnes ne participant pas à des activités physiques (Wessel, Wentz et Markle, 2011). Chez les personnes ayant subi une amputation, une revue systématique a constaté des effets psychologiques bénéfiques des activités réalisées tant en groupe qu'individuellement, notamment en améliorant la perception qu'ont les athlètes d'eux-mêmes (Bragaru et al., 2011). De plus, une plus grande confiance en soi a également été observée chez des athlètes ayant une tétraplégie qui pratiquent le rugby (Litchke et al., 2012). Ces personnes rapportent entre autres mieux s'adapter aux différentes situations et avoir davantage confiance en elles lors de situations sociales.

Une meilleure perception de soi est également exprimée par les personnes ayant une déficience intellectuelle et participant à des activités physiques. Une revue systématique (Hutzler et Korsensky, 2010) rapporte un plus grand sentiment de compétence et une plus haute estime de soi chez ces personnes, qui pourrait s'expliquer par l'amélioration des habiletés physiques (Castagno, 2001). Toutefois, une étude rapporte une absence d'effet observée sur la perception de soi suite à la participation à des activités physiques (Oriel, George et Blatt, 2008). Dans le cadre de cette étude, un programme d'exercices a été instauré auprès de jeunes ayant une déficience intellectuelle et visait à leur faire réaliser différentes activités à raison d'une séance par semaine. Selon les auteurs, les changements minimaux observés pourraient être dus au fait que l'intensité du programme était trop faible et devrait ultimement être d'une fréquence supérieure à une séance par semaine.

Les relations interpersonnelles semblent également bénéficier de la participation à des activités physiques compétitives chez les personnes ayant une incapacité physique (Briere III et Siegle, 2008; Wessel, Wentz et Markle, 2011). Chez les personnes ayant subi une amputation, une revue systématique (Bragaru et al., 2011) rapporte que les activités sportives réalisées en groupe constitueraient une occasion permettant le développement de nouvelles relations sociales. Des résultats semblables sont rapportés auprès des personnes ayant une paralysie cérébrale (Barfield et Malone, 2013) où la participation à du soccer compétitif est vue comme une occasion de passer du temps avec ses amis et autres personnes appréciées. II serait donc plausible de penser que les activités sportives seraient vécues comme un lieu d'échange et de rassemblement entre les participants.

\section{Le travail}

Chez les personnes ayant une incapacité physique, la possibilité d'exercer un travail dans sa communauté a été rapportée comme un facteur pouvant améliorer la qualité de vie générale des personnes en permettant un sentiment de contrôle perçu (Pawłowska-Cyprysiak, Konarska et Żołnierczyk-Zreda, 2015). Chez les personnes ayant une déficience intellectuelle, des effets positifs du travail sont rapportés, tant en milieu protégé qu'ordinaire, c'est-à-dire un environnement de travail qui n'est pas spécifique aux personnes ayant des incapacités. Le travail en environnement non protégé semble favoriser une meilleure qualité de vie (Kober et Eggleton, 2005), notamment dans le cadre d'un soutien en emploi (Beyer et al., 2010). En effet, les personnes exerçant un travail dans ce type 
d'environnement vivraient davantage un sentiment d'empowerment ce qui pourrait influencer le niveau de qualité de vie perçu. Le travail en environnement protégé, c'est-à-dire au sein d'un environnement adapté aux personnes ayant des incapacités, est lui aussi associé à des bienfaits. Par exemple, les personnes travaillant dans une micro-entreprise rapportent une augmentation de leur qualité de vie au travail par rapport à avant leur entrée dans la micro-entreprise (Conroy, Ferris et Irvine, 2010). Toutefois, tel que le mentionnent Kober et Eggleton (2005), il est possible que les personnes ayant préalablement un plus haut niveau d'empowerment choisissent davantage d'exercer un emploi dans un milieu de travail non protégé. Certaines caractéristiques personnelles des participants pourraient donc potentiellement nous renseigner et expliquer en partie leur présence dans un type ou un autre des environnements de travail.

Le milieu du travail est lui aussi considéré comme un moteur important du développement de relations sociales chez les personnes en situation de handicap. Lamichhane (2012) souligne que les travailleurs ayant une déficience visuelle, auditive ou motrice rapportent une plus grande inclusion sociale et un plus grand nombre d'amis depuis qu'ils exercent un travail. Les contacts sociaux qui ont lieu au travail s'étendraient également à l'extérieur de celui-ci. Le travail serait donc vécu comme un lieu d'échange qui permettrait aux personnes de développer un réseau de soutien qui s'étend au-delà de la période travaillée. Comme l'indique Lamichhane (2012), lorsque des personnes ayant une incapacité socialisent avec leurs collègues, elles renforcent par le fait même leur visibilité dans la communauté, ce qui aide à réduire les préjugés et la discrimination qu'elles peuvent vivre.

\section{Les loisirs}

Les activités de loisirs réalisées par les personnes ayant une incapacité physique sont associées à des conséquences positives sur leur qualité de vie. Des interventions ont été effectuées afin de faire vivre des périodes de loisirs, tant individuelles qu'en groupe, à des personnes ayant une déficience physique, notamment des personnes paraplégiques. Les activités de loisirs favoriseraient la qualité de vie, une plus grande appartenance sociale et un plus haut niveau d'engagement psychologique chez les personnes qui les pratiquent. Ce type d'activité favoriserait de plus la réflexion individuelle des personnes en ce qui concerne leur vie ainsi que leurs valeurs personnelles (Daniel et Manigandan, 2005; King et al., 2014).

Chez les personnes ayant une déficience intellectuelle, deux études se sont intéressées aux impacts psychosociaux de la réalisation de loisirs et ont toutes deux observé que la participation à des loisirs ne prédisait pas la qualité de vie des participants (Badia et al., 2013; Miller et Chan, 2008). Dans ces études, les personnes devaient préciser les loisirs qu'elles ont pratiqués au cours des deux dernières semaines (Miller et Chan, 2008) ou de la dernière année (Badia et al., 2013). Selon Miller et Chan (2008), les loisirs ne prédisent pas la satisfaction de vie des personnes ayant une déficience intellectuelle, car il est possible qu'elles aient de la difficulté à trouver une activité leur permettant précisément de ressentir un sentiment de satisfaction suffisant.

\section{L'école}

Une seule étude s'est intéressée aux impacts de la fréquentation du système scolaire chez les jeunes ayant des incapacités variées suite à la sortie de l'école secondaire (Repetto et al., 2011). Dans cette étude, des étudiants avec et sans incapacités devaient remplir un sondage dans lequel ils rapportaient 
différentes informations reliées à leur passage à l'école secondaire. Comparativement aux participants sans incapacité, les personnes en situation de handicap rapportent davantage savoir ce qu'elles veulent faire dans le futur et comment réaliser leurs rêves. II semble donc plausible de croire que l'école serait un vecteur important de la transition vers la vie adulte en procurant de la motivation et de la confiance aux jeunes.

\section{La thérapie par la musique.}

Le dernier type d'activité répertorié comme ayant un impact sur la qualité de vie est la thérapie par la musique, réalisée auprès de jeunes adultes vivant des problèmes de santé mentale importants (Cathro et Devine, 2012). Le but de cette thérapie occupationnelle était d'aider les jeunes à faire face à des difficultés telles que l'isolement social, un faible répertoire d'activités, de même que des symptômes spécifiques à leur maladie. Dans le cadre de cette thérapie, les participants devaient apprendre à jouer de différents instruments et bâtissaient ensemble un répertoire musical de leur cru. Les personnes qui y ont participé ont généralement rapporté une amélioration de leur humeur et de leur motivation de même qu'une participation plus active dans la vie sociale à la suite de cette thérapie. La thérapie par la musique semble être vue par les participants comme une occasion d'apprentissage et de socialisation leur permettant une plus grande valorisation personnelle.

\section{Discussion}

Le but de cette recension était de documenter les effets psychosociaux des activités visant à soutenir les jeunes adultes en situation de handicap. Les activités répertoriées sont le sport, les loisirs, le travail, l'école et la thérapie par la musique. Les résultats démontrent majoritairement des bénéfices psychosociaux importants liés à la participation à ces activités. Des effets sont constatés notamment sur la qualité de vie générale de même que sur la perception de soi et les relations interpersonnelles des individus. L'augmentation de la qualité de vie des personnes pourrait être influencée par une meilleure santé psychologique. De fait, Sweet, Martin Ginnis et Tomasone (2013) mentionnent que les symptômes dépressifs et l'indépendance fonctionnelle pourraient influencer la relation entre la participation à des loisirs sportifs et la qualité de vie chez les personnes ayant une lésion de la moelle épinière. De plus, une récente méta-analyse (Rosenbaum et al., 2014) met en lumière la réduction des symptômes dépressifs associée à la participation à des sports chez les personnes sans incapacité. Finalement, les activités permettant d'interagir avec autrui, que ce soit par le biais de loisirs, d'activités sportives ou encore par l'entremise d'un travail, permettent aux personnes de développer de nouvelles relations et de consolider leur réseau social.

Nous avons également constaté que le contexte adéquat de réalisation des différentes activités peut varier selon la situation de handicap vécue par la personne. Par exemple, dans le cadre d'activités physiques, un contexte compétitif a des retombées positives chez les jeunes adultes vivant avec une incapacité physique. Toutefois, nous avons observé qu'il est associé à une hausse de la comparaison sociale chez les personnes ayant une déficience intellectuelle ce qui est lié à une baisse d'estime de soi. II faut toutefois noter que plusieurs études ont été réalisées auprès de personnes ayant une déficience physique qui sont également des athlètes (Fiorilli et al., 2013; Groff, Lundberg et Zabriskie, 2009; Hutzler, Chacham-Guber et Reiter, 2013; Litchke et al., 2012; Wessel, Wentz et Markle, 2011) alors que la majorité des études concernant les activités physiques chez les personnes ayant une déficience intellectuelle portent sur des interventions visant à augmenter leur participation à des activités 
physiques (Bartlo et Klein, 2011; Carraro et Gobbi, 2014; Ogg-Groenendaal, Hermans et Claessens, 2014).

\section{Limites}

Certaines limites sont toutefois à prendre en compte dans cette étude. En premier lieu, il est possible que les résultats obtenus puissent ne pas s'appliquer à tous les types d'incapacité abordés. De fait, plusieurs études posent des conclusions auprès de personnes ayant différents types d'incapacité et les résultats y sont généralisés à l'ensemble des personnes. Finalement, il est possible de constater une sous-représentation des personnes ayant des incapacités visuelles et auditives dans les études. II importe donc d'être prudent dans la généralisation des résultats obtenus envers les personnes vivant ces types particuliers d'incapacité.

\section{Implications pratiques}

Les données disponibles indiquent une hausse de la qualité de vie découlant de la participation à des activités comme le sport, les loisirs, le travail, l'école et la thérapie par la musique. Elle semble être surtout favorisée par les activités permettant une certaine compétition entre les participants ayant des incapacités physiques. Hutzler et Korsensky (2010) ont aussi émis la recommandation que l'environnement où les personnes ayant une déficience intellectuelle réalisent leurs activités physiques devrait viser un climat de collaboration plutôt que de compétition. Les loisirs de groupe sont perçus comme un moment de rencontre et de plaisir entre amis et le travail, tant en environnement protégé que non protégé, favorise le développement de nouvelles relations qui contribueront sans doute à fournir un soutien social aux personnes vivant avec une incapacité. Finalement, l'apprentissage est également une composante clé des activités en permettant la valorisation des personnes dans les activités réalisées (Cathro et Devine, 2012). En conclusion, il importe de poser une attention particulière au contexte de réalisation des activités en s'interrogeant sur les limitations particulières des personnes de même qu'en veillant à instaurer un climat de collaboration où un réseau social pourra être tissé entre les membres. Cela peut être réalisé par le biais d'activités effectuées en groupe, que ce soit des loisirs, des activités physiques ou par la présence sur le marché du travail. À la lumière de la globalité des précédents résultats, il est possible d'avancer l'importance d'instaurer un contexte inclusif permettant aux personnes d'occuper une place au cœur de la communauté. 
Figure 1. Processus de sélection des articles

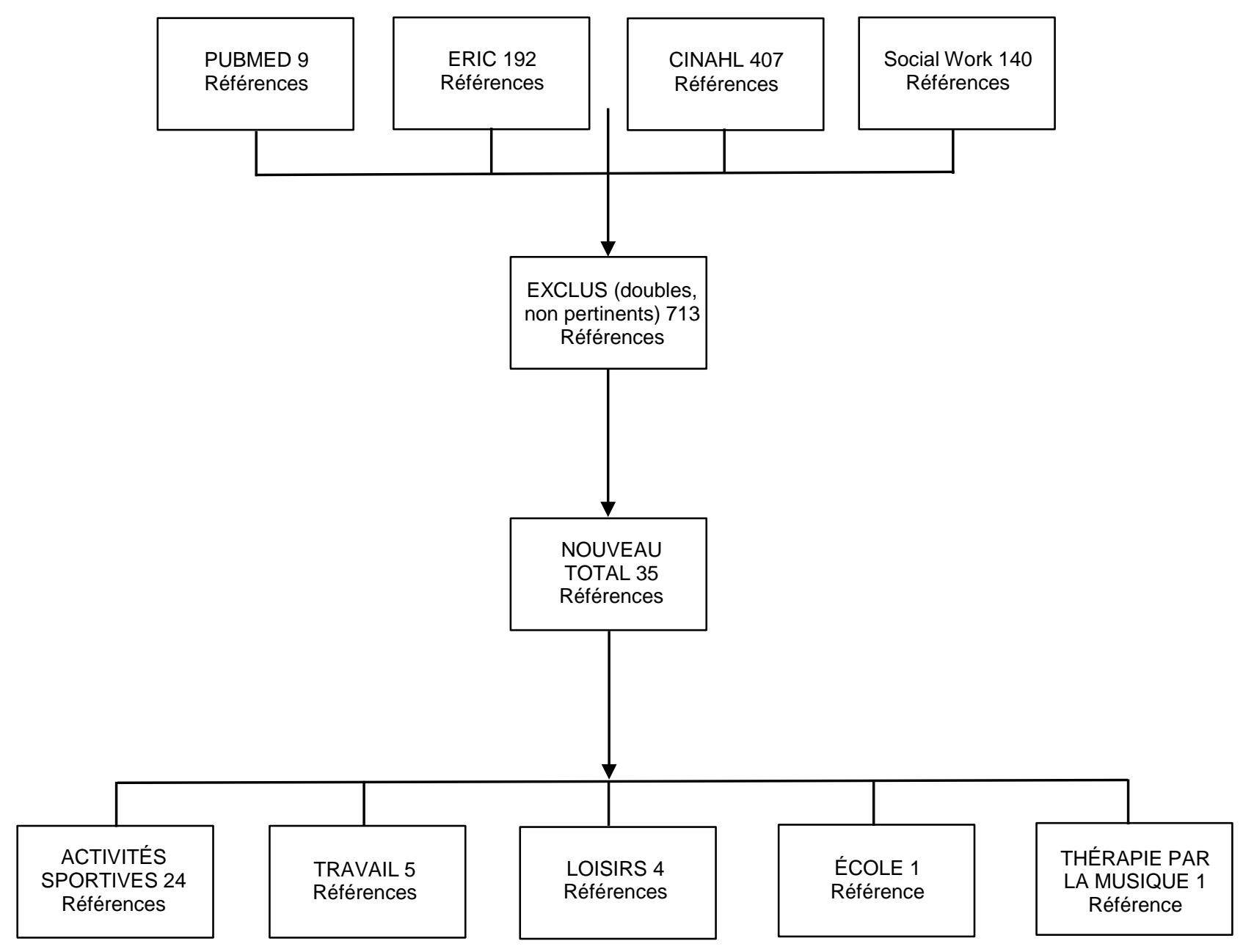

Note. D'autres articles jugés pertinents et identifiés à partir de la liste de références des articles consultés ont également été inclus. 
Figure 2. Activités propices au développement de la qualité de vie, d'une perception de soi positives et des relations interpersonnelles
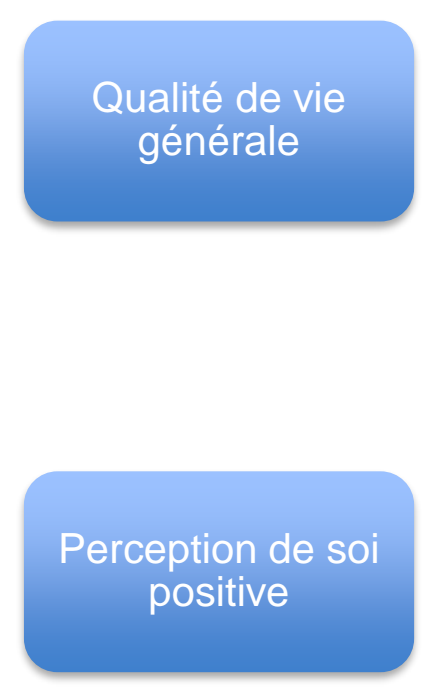

Relations interpersonnelles
- Les activités physiques devraient miser sur un contexte de réalisation compétitif qui est associé à des bienfaits comme une meilleure santé mentale et une plus grande qualité de sommeil chez les personnes ayant une déficience physique.

- Le travail, en milieu protégé et non protégé, favoriserait le sentiment de contrôle perçu chez les participants.

- Les loisirs permettent le développement de relations sociales qui apporteront un soutien important dans la vie des personnes.

- Un contexte de compétition sportive est associé à un plus grand sentiment d'indépendance, de la confiance en soi ainsi qu'une perception de soi positive chez les personnes ayant une déficience physique.

- Toutefois, la compétition semble mener à une baisse d'estime de soi chez les personnes ayant une déficience intellectuelle en les exposant à une plus grande comparaison sociale.

- Les activités physiques sont vécues comme des moments de coopération et d'échange entre les participants.

- Le fait d'exercer un travail permet une plus grande inclusion sociale, une augmentation du nombre d'amis et le développement de relations qui s'étendent à l'extérieur du milieu de travail.

FALESCHINI, Sabrina

BOUCHER, Normand

\section{RÉFÉRENCES}

Badia, M., M.B. Orgaz, M.A. Verdugo, A.M.Ullan et M. Martinez (2013). « Relationships between Leisure Participation and Quality of Life of People with Developmental Disabilities », Journal of Applied Research in Intellectual Disabilities, vol. 26, $n^{\circ} 6$, p. 533-545.

Barfield, J.P., et L.A. Malone (2013). «Perceived exercise benefits and barriers among power wheelchair soccer players », Journal of Rehabilitation Research and Development, vol. 50, $\mathrm{n}^{\circ} 2$, p. 231-238. doi: 10.1682/jrrd.2011.12.0234 
Bartlo, P., et P.J. Klein (2011). "Physical Activity Benefits and Needs in Adults With Intellectual Disabilities: Systematic Review of the Literature ", American Journal on Intellectual and Developmental Disabilities, vol. 116, $\mathrm{n}^{\circ}$ 3, p. 220-232.

Beyer, S., T. Brown, R. Akandia et M. Rapley (2010). «A Comparison of Quality of Life Outcomes for People with Intellectual Disabilities in Supported Employment, Day Services and Employment Enterprises ", Journal of Applied Research in Intellectual Disabilities, vol. 23, n 3, p. 290-295.

Bragaru, M., R. Dekker, J.H.B. Geertzen et P.U. Dijkstra (2011). "Amputees and Sports A Systematic Review », Sports Medicine, vol. 41, n 9, p. 721-740.

Brasile, F. M. (1992). «Inclusion: A developmental perspective. A rejoinder to "Examining the concept of reverse integration" », Adapted Physical Activity Quarterly, vol. 9, p. 293-304.

Briere III, D.E., et D. Siegle (2008). "The Effects of the Unified Sports Basketball Program on Special Education Students' Self-Concepts: Four Students' Experiences ", TEACHING Exceptional Children Plus, vol. 5, no 1, p. 1-12.

Buffart, L.M., R.J. van den Berg-Emons, J. van Meeteren, H.J. Stam et M.E. Roebroeck (2009). "Lifestyle, participation, and health-related quality of life in adolescents and young adults with myelomeningocele », Developmental Medicine and Child Neurology, vol. 51, n 11, p. 886-894. doi: 10.1111/j.1469-8749.2009.03293.x

Carraro, A., et E. Gobbi (2014). " Exercise intervention to reduce depressive symptoms in adults with intellectual disabilities ", Perceptual and Motor Skills, vol. 119, $n^{\circ} 1$, p. 1-5. doi: 10.2466/06.15.PMS.119c17z4

Castagno, K.S. (2001). "Special Olympics Unified Sports: Changes in Male Athletes During a Basketball Season », Adapted Physical Activity Quartely, vol. 18, p. 193-206.

Cathro, M., et A. Devine (2012). « Music therapy and social inclusion », Mental Health Practice, vol. 16, $n^{\circ} 1$, p. 33-36.

Collins, B., et P. O'Mahony (2015). " Physically Disabled Adults' Perceptions of Personal Autonomy: Impact on Occupational Engagement », OTJR: Occupation, Participation and Health, vol. 35, $\mathrm{n}^{\circ} 3$, p. $160-168$. doi: $10.1177 / 1539449215575264$

Conroy, J.W., C.S. Ferris, et R. Irvine (2010). « Microenterprise Options for People With Intellectual and Developmental Disabilities: An Outcome Evaluation », Journal of Policy and Practice in Intellectual Disabilities, vol. 7, $\mathrm{n}^{\circ}$ 4, p. 269-277.

Daniel, A., et C. Manigandan (2005). « Efficacy of leisure intervention groups and their impact on quality of life among people with spinal cord injury ", International Journal of Rehabilitation Research, vol. $28, n^{\circ} 1$, p. $43-48$. 
Di Cagno, A., E. Iuliano, G. Aquino, G. Fiorilli, C. Battaglia, A. Giombini, et G. Calcagno (2013). «Psychological well-being and social participation assessment in visually impaired subjects playing Torball: a controlled study », Research in Developmental Disabilities, vol. 34, nº 4, p. 1204-1209. doi: 10.1016/j.ridd.2012.11.010

Dijkers, M.P.J.M. (2005). «Quality of life of individuals with spinal cord injury: A review of conceptualization, measurement, and research findings », Journal of Rehabilitation Research and Development, vol. 42, n 3, p. 87-110.

Fiorilli, G., E. Iuliano, G. Aquino, C. Battaglia, A. Giombini, G. Calcagno et A. di Cagno (2013). « Mental health and social participation skills of wheelchair basketball players: a controlled study », Research in Developmental Disabilities, vol. 34, n 11, p. 3679-3685. doi: 10.1016/j.ridd.2013.08.023

Fougeyrollas, P. (2010). La funambule, le fil et la toile. Transformations réciproques du sens du handicap, Québec, Presses de l'Université Laval.

George, A.L., et C. Duquette (2006). «The Psychosocial Experiences of a Student with Low Vision », Journal of Visual Impairment \& Blindness, vol. 100, nº 3, p. 152-163.

Giacobbi Jr, P.R., M. Stancil, B. Hardin et L. Bryant (2008). «Physical Activity and Quality of Life Experienced by Highly Active Individuals With Physical Disabilities », Adapted Physical Activity Quarterly, vol. 25, n³, p.189-207.

Groff, D.G., N.R. Lundberg et R.B. Zabriskie (2009). "Influence of adapted sport on quality of life: perceptions of athletes with cerebral palsy », Disability \& Rehabilitation, vol. 31, n 4, p. 318-326. doi: $10.1080 / 09638280801976233$

Hutzler, Y., A. Chacham-Guber et S. Reiter (2013). "Psychosocial effects of reverse-integrated basketball activity compared to separate and no physical activity in young people with physical disability », Research in Developmental Disabilities, vol. 34, $\mathrm{n}^{\circ} 1$, p. 579-587. doi: 10.1016/j.ridd.2012.09.010

Hutzler, Y., et O. Korsensky (2010). « Motivational correlates of physical activity in persons with an intellectual disability: a systematic literature review », Journal of Intellectual Disability Research, vol. 54, nº 9, p. 767-786. doi: 10.1111/j.1365-2788.2010.01313.x

King, G., B. Batorowicz, P. Rigby, M. Pinto, L. Thompson et F. Goh (2014). "The leisure activity settings and experiences of youth with severe disabilities ", Developmental Neurorehabilitation, vol. 17, no 4, p. 259-269. doi: 10.3109/17518423.2013.799244

Kober, R., et I.R.C. Eggleton (2005). "The effect of different types of employment on quality of life », Journal of Intellectual Disability Research, vol. 49, n 10, p. 756-760.

Lamichhane, K. (2012). «Employment situation and life changes for people with disabilities: evidence from Nepal », Disability \& Society, vol. 27, n 4, p. 471-485. doi: 10.1080/09687599.2012.659462 
Landry, L., et C. Boucher (2011). L'arrimage de la Transition de l'École à la Vie Active (TEVA) au Parcours de Formation Axée sur l'Emploi (PFAE), Alma (QC), Service de soutien et d'expertise du Saguenay-Lac-Saint-Jean.

Litchke, L.G., J.S. Hodges, E.A. Schmidt, L.K. Lloyd, E. Payne et C.J. Russian (2012). «Personal Meaning of Wheelchair Rugby Participation by Five Male Athletes ", Therapeutic Recreation Journal, vol. $46, n^{\circ} 1$, p. $26-41$.

Martin, J.J. (2008). "Multidimensional Self-Efficacy and Affect in Wheelchair Basketball Players ", Adapted Physical Activity Quarterly, vol. 25, nº 4, p. 275-288.

Miller, S.M., et F. Chan (2008). "Predictors of life satisfaction in individuals with intellectual disabilities ", Journal of Intellectual Disability Research, vol. 52, n 12, p. 1039-1047. doi: 10.1111/j.1365-2788.2008.01106.x

Nasuti, G., et V.A. Temple (2010). « The risks and benefits of snow sports for people with disabilities: a review of the literature ", International Journal of Rehabilitation Research, vol. 33, n 3, p. 193-198. doi: 10.1097/MRR.0b013e32833638a5

National Organization on Disability (2010). The ADA, 20 years later, New York, Harris Interactive.

Ninot, G., J. Bilard et D. Delignieres (2005). « Effects of integrated or segregated sport participation on the physical self for adolescents with intellectual disabilities ", Journal of Intellectual Disability Research, vol. 49, no 9, p. 682-689. doi: 10.1111/j.1365-2788.2005.00407.x

Office des personnes handicapées du Québec (2003). La transition de l'école à la vie active. Québec, Gouvernement du Québec.

Office des personnes handicapées du Québec (2013). Consultation sur le livre vert : Le goût et le plaisir de bouger : vers une politique nationale du sport, du loisir et de l'activité physique, Drummondville (QC), Office des personnes handicapées du Québec.

Ogg-Groenendaal, M., H. Hermans et B. Claessens (2014). "A systematic review on the effect of exercise interventions on challenging behavior for people with intellectual disabilities ", Research in Devolpmental Disability, vol. 35, nº 7, p. 1507-1517. doi: 10.1016/j.ridd.2014.04.003

Oriel, K.N., C.L. George et P.J. Blatt (2008). « The Impact of a Community Based Exercice Program in Children and Adolescents with Disabilities: A Pilot Study ", Physical Disabilities: Education and Related Services, vol. 27, no 1, p. 5-20.

Pawłowska-Cyprysiak, K., M. Konarska et D. Żołnierczyk-Zreda (2015). « Self-Perceived Quality of Life of People With Physical Disabilities and Labour Force Participation ", International Journal of Occupational Safety and Ergonomics, vol. 19, ${ }^{\circ} 2, \quad$ p. 185-194. doi: 10.1080/10803548.2013.11076977 
Repetto, J.B., S.P. McGorray, H. Wang, M. Podmostko, W.D. Andrews, J. Lubbers et S. Gritz (2011). «The High School Experience: What Students With and Without Disabilities Report as They Leave School », Career Development and Transition for Exceptional Individuals, vol. 34, n 3, p. 142-152. doi: $10.1177 / 0885728811414699$

Rosenbaum, S., A. Tiedemann, C. Sherrington, J. Curtis et P.B. Ward (2014). «Physical activity interventions for people with mental illness: a systematic review and meta-analysis », Journal of Clinical Psychiatry, vol. 75, n 9, p. 964-974. doi: 10.4088/JCP.13r08765

Semerjian, T. Z., S.M. Montague, J.F. Dominguez et R.D. de Leon (2005). « Enhancement of Quality of Life and Body Satisfaction Through the Use of Adapted Exercise Devices for Individuals with Spinal Cord Injuries », Topics in Spinal Cord Injury Rehabilitation, vol. 11, n², p. 95-108.

Schalock, R.L., G.S. Bonhamb, et M.A. Verdugoc (2008). « The conceptualization and measurement of quality of life: Implications for program planning and evaluation in the field of intellectual disabilities », Evaluation and Program Planning, vol. 31, $\mathrm{n}^{\circ}$ 2, p. 181-190.

Smetana, J.G. (2011). «Coordinations and Change in Social Development », in Adolescents, Families, and Social Development: How Teens Construct Their Worlds, Malden (MA), Wiley-Blackwell.

Statistique Canada (2013). L'enquête canadienne sur l'incapacité. Ottawa (ON), Gouvernement du Canada.

Sung, C., C.Y. Chiu, E.J. Lee, J. Bezyak, F. Chan et V. Muller (2012). «Exercise, Diet, and Stress Management as Mediators Between Functional Disability and Health-Related Quality of Life in Multiple Sclerosis », Rehabilitation Counseling Bulletin, vol. 56, $\mathrm{n}^{\circ} 2$, p. 85-95. doi: $10.1177 / 0034355212439899$

Sweet, S.N., K.A. Martin Ginis et J.R. Tomasone (2013). «Investigating intermediary variables in the physical activity and quality of life relationship in persons with spinal cord injury », Health Psychology, vol. 32, nº 8 , p. 877-885. doi: 10.1037/a0032383

Tsimaras, V.K., et E.G. Fotiadou (2004). « Effect of training on the muscle strength and dynamic ability of adults with Down syndrome », Journal of Strength and Conditioning Research, vol. 18, $\mathrm{n}^{\circ} 2$, p. 343-347.

Werner, S. (2015). “Athletes', parents', and siblings' experiences from the Special Olympics World Games », Journal of Intellectual and Developmental Disability, vol. 40, $\mathrm{n}^{\circ} 2$, p. 167-178. doi: $10.3109 / 13668250.2015 .1010148$

Wessel, R.D., J. Wentz et L.L. Markle (2011). «Power Soccer: Experiences of Students Using Power Wheelchairs in a Collegiate Athletic Club », Journal of Postsecondary Education and Disability, vol. 24, n 3 , p. 147-159. 
Yazicioglu, K., F. Yavuz, A.S. Goktepe et A.K. Tan (2012). «Influence of adapted sports on quality of life and life satisfaction in sport participants and non-sport participants with physical disabilities ", Disability and Health Journal, vol. 5, nº 4, p. 249-253. doi: 10.1016/j.dhjo.2012.05.003 